\title{
O vídeo-EEG dia no diagnóstico de eventos paroxísticos na infância
}

\author{
The diagnostic value of short-term video-EEG monitoring in childhood
}

\author{
Alessandra Freitas ${ }^{1}$, Lia A. Fiore ${ }^{2}$, Gary Gronich ${ }^{3}$, Kette D. Valente ${ }^{4}$
}

\section{Resumo}

Objetivo: este estudo tem como objetivo investigar o valor do vídeo-EEG dia numa população pediátrica, com queixas clínicas diversas, verificando os benefícios e as limitações deste método.

Casuística e métodos: um protocolo prospectivo, desenvolvido na Universidade de São Paulo, analisou 38 pacientes consecutivos (quatro meses a 17 anos; média 6,9 anos). Todos os pacientes foram encaminhados para elucidação do seu quadro clínico. Estes foram classificados, segundo sua queixa clínica principal, em: dúvidas sobre a classificação das crises/ síndromes epilépticas em 22 pacientes (grupo I); diagnóstico diferencial entre eventos epilépticos e não epilépticos em 8 (grupo II); e diagnóstico diferencial entre declínio cognitivo e estado de mal epiléptico (EME) não convulsivo em 8 pacientes (grupo III).

Resultados: episódios clínicos foram registrados em 36 pacientes $(94,7 \%)$. No grupo I, as crises epilépticas foram reclassificadas em $11 / 22(50 \%)$ pacientes e confirmadas em oito $(36,4 \%)$. Deste grupo, um paciente apresentou distúrbio do sono, e dois não apresentaram eventos clínicos durante a monitorização. A classificação sindrômica foi modificada em nove pacientes $(40,9 \%)$. No grupo II, quatro pacientes $(50 \%)$ apresentaram eventos epilépticos. A deterioração cognitiva estava associada com EME em cinco crianças $(62,5 \%)$ do grupo III. Mudanças na conduta terapêutica e diagnóstica, como consequiência da monitorização, ocorreram em 21/38 $(55,3 \%)$ pacientes.

Conclusão: em nossa série, o vídeo-EEG dia estabeleceu o diagnóstico na maioria dos pacientes, relacionando os dados clínicos com os eletrencefalográficos. Este procedimento foi bem tolerado pelas crianças, incluindo lactentes e aquelas com doenças psiquiátricas.

J Pediatr (Rio J) 2003;79(3):259-64: epilepsia, monitorização, classificação.

\begin{abstract}
Objective: the objective of this study was to investigate the value of short-term video-EEG monitoring in a pediatric population with distinct clinical complaints in order to verify the benefits and limitations of this procedure.
\end{abstract}

Patients and methods: a prospective protocol, developed in the University of São Paulo, analyzed 38 consecutive patients (age ranging from 4 months to 17 years; mean 6.9 years). All patients were referred in order to establish the diagnosis. The patients were divided in the following groups according to the main clinical complaint: doubts about seizure/syndromic classification (Group I, $\mathrm{n}=22$ ); differential diagnosis with non-epileptic events (Group II, $\mathrm{n}=8$ ) and differential diagnosis between cognitive decline and status epilepticus (Group III, $\mathrm{n}=8$ ).

Results: clinical episodes were observed in 36 patients $(94.7 \%)$. In group I, seizures were reclassified in 11/22 (50\%) patients and confirmed in eight (36.4\%). One patient presented a sleep disorder and two did not present clinical events during monitoring. Syndromic classification was modified in nine $(40.9 \%)$. In group II, four patients $(50 \%)$ presented epileptic seizures; two had movement disorders and two, non-epileptic events. The cognitive deterioration was correlated with non-convulsive status epilepticus in five children $(62.5 \%)$ of group III. Alterations of therapeutic and/or diagnostic approaches, as a consequence of monitoring, were performed in $21 / 38(55.3 \%)$ patients.

Conclusion: in our series, short-term video-EEG monitoring established a reliable diagnosis in most patients due to correlation between clinical and EEG data. This procedure was well tolerated by children, including infants and those with psychiatric disorders.

J Pediatr (Rio J) 2003;79(3):259-64: epilepsy, monitoring, classification.

1. Médica colaboradora do Lab. de Neurofisiologia Clínica do Instituto de Psiquiatria do HC-FMUSP. Pós-graduanda do Instituto da Criança do HC-FMUSP.

2. Médica assistente do Lab. de Neurofisiologia Clínica do Instituto de Psiquiatria do HC-FMUSP. Mestre em Medicina pela Universidade de São Paulo.

3. Médico assistente do Lab. de Neurofisiologia Clínica do Instituto de Psiquiatria do HC-FMUSP.

4. Médica assistente do Lab. de Neurofisiologia Clínica do Instituto de Psiquiatria do HC-FMUSP. Doutora em Neurologia pela Universidade de São Paulo.

Artigo submetido em 27.11.02, aceito em 31.01.03. 


\section{Introdução}

O vídeo-EEG é um método diagnóstico não invasivo, caracterizado pelo registro do eletroencefalograma (EEG) concomitante à gravação de um evento clínico, fornecendo a correlação inequívoca entre estes dados. Desta forma, um evento paroxístico assistido e relatado por familiares ou pelo paciente pode ser documentado, e sua natureza, epiléptica ou não, pode ser determinada. Uma indicação importante deste tipo de procedimento inclui o diagnóstico diferencial entre eventos epilépticos e não epilépticos (síncopes, arritmias cardíacas, distúrbios do sono e distúrbios psiquiátricos, entre outros). Outras condições nas quais o vídeo-EEG é recomendado são a classificação de síndromes e crises epilépticas, a determinação da zona epileptogênica na investigação pré-cirúrgica para epilepsia, a determinação da freqüência das crises, e a resposta às intervenções terapêuticas ${ }^{1-11}$.

O vídeo-EEG pode ser realizado com a internação do paciente e a permanência do mesmo sob monitorização contínua dentro de uma unidade adequada às suas necessidades, onde seus eventos serão registrados durante 24 horas, por um período de tempo que, em média, varia de três a sete dias. Esta forma de monitorização, classicamente usada, é denominada de monitorização prolongada. A monitorização com vídeo-EEG, sem que haja a hospitalização do paciente e com uma permanência mais breve (4 a 12 horas), é denominado de vídeo-EEG dia, e tem como um de seus objetivos primordiais minimizar o desconforto da monitorização prolongada.

O vídeo-EEG prolongado, embora preste uma contribuição inegável na elucidação diagnóstica de eventos paroxísticos, apresenta algumas inconveniências, como a hospitalização prolongada, o desconforto da permanência prolongada com eletrodos colados ao couro cabeludo (através de colódio), assim como os custos elevados deste procedimento $^{4}$

A população pediátrica é a que mais se beneficia com o registro videoeletrencefalográfico, tendo em vista a peculiaridade das crises epilépticas na infância, a incapacidade destes pacientes em descrever fenômenos subjetivos e a dificuldade de caracterizar a natureza de um evento, mesmo quando este é assistido por um profissional. Entretanto, as crianças representam um grande desafio para a realização do vídeo-EEG prolongado, tendo em vista a hospitalização como um importante fator estressor, assim como a dificuldade da criança em compreender o procedimento imposto. Há poucas publicações sobre o valor diagnóstico do vídeoEEG de curta duração com pacientes pediátricos ambulatoriais (vídeo-EEG dia $<24 h$ ) ${ }^{8,9}$. Por causa da escassez de estudos, há muitas estimativas sobre a sensibilidade do método, que difere sobremaneira entre os diversos estudos $(55 \mathrm{a} 83 \%)^{8-10}$, devido às variações das populações estudadas e do tipo de indicação.

O objetivo deste estudo prospectivo é investigar o valor do vídeo-EEG dia numa população pediátrica, com queixas clínicas diversas, verificando os benefícios e as limitações deste método.

\section{Pacientes e métodos \\ População estudada}

Um protocolo prospectivo foi desenvolvido no laboratório de neurofisiologia clínica do Hospital das Clínicas da FMUSP, no período de maio de 2000 a março de 2002, e foram analisados 38 pacientes consecutivos (idade entre quatro meses e 17 anos - média de 6,9 anos; mediana de seis anos). Todos os pacientes foram encaminhados de centros terciários de neurologia e pediatria, para elucidação clínica, e foram categorizados em três grupos, de acordo com a queixa principal. O grupo I foi formado por 22 pacientes, com forte suspeita clínica de epilepsia, encaminhados para a melhor classificação das crises e/ou síndromes epilépticas apresentadas.

O grupo II foi constituído por oito pacientes com suspeita clínica de eventos não epilépticos. Um denominador comum entre estes pacientes foi a presença de comprometimento da interação com o meio, pela patologia de base (presença de distúrbio psiquiátrico em seis pacientes e deficiência mental grave nos demais), o que representou a principal dificuldade para o diagnóstico clínico através da entrevista com os familiares e observação direta do evento apresentado pelo paciente.

O grupo III, formado por oito pacientes com atraso grave do desenvolvimento neuropsicomotor (quatro com síndrome de Angelman, um com síndrome de Rett, um com atrofia óptica de Leber, um com síndrome de Alpers e um com suspeita de doença metabólica, em investigação). Estes pacientes apresentaram declínio cognitivo súbito, caracterizado, em três crianças, por perda de aquisições prévias e, nos outros, por períodos prolongados de irresponsividade.

Em 11 pacientes $(28,9 \%)$, mais de uma queixa clínica esteve associada, e os pacientes foram classificados de acordo com a razão mais importante para a realização do procedimento.

\section{Protocolo de estudo}

O protocolo deste estudo incluiu a revisão dos EEGs prévios e anamnese e exame físico dirigidos para verificar a necessidade de adição de eletrodos, registros poligráficos, modificação de ciclo circadiano e/ou outros métodos de ativação.

\section{Avaliação vídeo-EEG}

Durante a monitorização, eletrodos de escalpo foram posicionados de acordo com o sistema 10-2012 e com adição de eletrodos adicionais [sistema 10-10], quando necessário. Durante a crise e logo após o término da mesma, todos os pacientes foram testados, visando verificar o nível 
de consciência e alterações neurológicas transitórias (por exemplo, funções de linguagem, motoras ou de memória). Os pacientes não necessitaram de hospitalização, e foram avaliados em estudos vídeo-EEG que variaram entre 4-12 horas de duração (média de 8 horas). Os registros foram obtidos em vigília, sonolência e sono.

\section{Revisão do vídeo-EEG}

Após a monitorização, os pacientes e/ou suas famílias reviram os eventos registrados. Caso estes registros não fossem reconhecidos pelos familiares como aqueles observados em casa, o exame era considerado inconclusivo, e o paciente convocado para um segundo registro. Se este segundo registro não fosse suficiente, o paciente era, então, encaminhado para uma monitorização vídeo-EEG prolongada. Posteriormente, os vídeos e os traçados eletrencefalográficos foram revistos por três dos autores, independentemente.

\section{Classificação dos eventos}

As crises e as síndromes epilépticas foram classificadas de acordo com a Liga Internacional Contra a Epilepsia $(\text { ILAE })^{13}$. Antes do início da monitorização, a classificação foi feita somente com base na história clínica e EEGs prévios. Os estudos de neuroimagem realizados também acrescentaram dados para a definição etiológica das crises epilépticas em sintomática, criptogênica ou idiopática.

\section{Resultados}

Episódios clínicos foram registrados em 36 de 38 pacientes (94,7\%), dentre os quais, 27 (71\%) nas primeiras 12 horas. Os outros onze pacientes necessitaram um segundo procedimento de 12 horas, o qual foi conclusivo em nove casos. Dos pacientes monitorizados, dez foram encaminhados à videomonitorização prolongada, para avaliação préoperatória.

Todos os 27 pacientes nos quais o registro de eventos se deu durante as primeiras 12 horas apresentavam história prévia de crises diárias, enquanto os restantes (11 pacientes) apresentavam eventos semanais.

\section{Grupo I}

Este grupo foi composto por 22 crianças (6 lactentes) com diagnóstico prévio de epilepsia em bases clínicas e eletrencefalográficas, exceto por um paciente que apresentava EEGs de rotina normais.

Somente $2 / 22$ pacientes $(9,1 \%)$, anteriormente mencionados, não apresentaram eventos clínicos durante a monitorização e foram encaminhados ao vídeo-EEG prolongado. Dos vinte pacientes que tiveram registro clínico, um paciente apresentou somente eventos não epilépticos. Nos outros, as crises epilépticas estavam erroneamente classificadas em 11 pacientes (50\%) (Figura 1). O principal erro

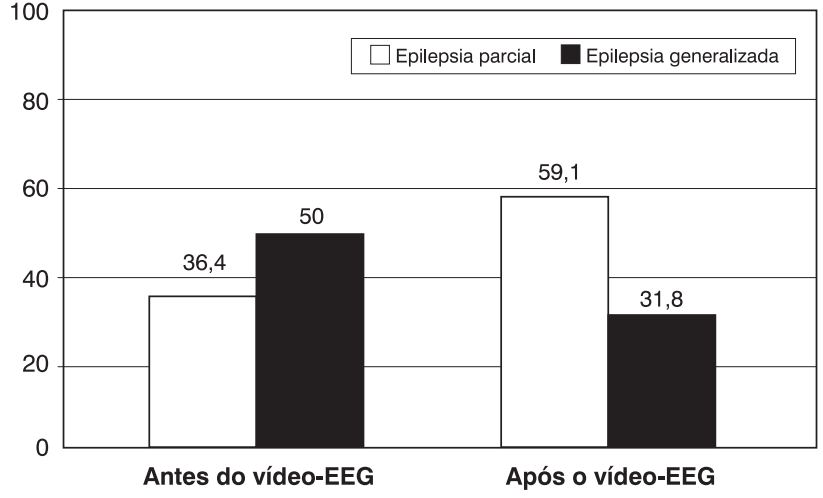

Figura 1 - Alteração da classificação das crises epilépticas após a realização do vídeo-EEG

diagnóstico ocorreu na classificação das crises parciais, seguidas de rápida generalização secundária, que foram previamente diagnosticadas como crises generalizadas (seis pacientes). Quanto à síndrome epiléptica, o vídeo-EEG confirmou o diagnóstico realizado através da história e EEG de rotina em onze (50\%) e levou à revisão do diagnóstico etiológico em nove pacientes (40,9\%) (Figura 2).

Em oito pacientes com epilepsia, havia a suspeita clínica do aparecimento de um novo tipo de crise epiléptica ou uma mudança semiológica das crises previamente existentes, sendo possível demonstrar, através do vídeo-EEG dia, que estes eventos não eram epilépticos em sete pacientes. Por outro lado, crises epilépticas sutis, imperceptíveis aos pais, foram documentadas em um paciente.

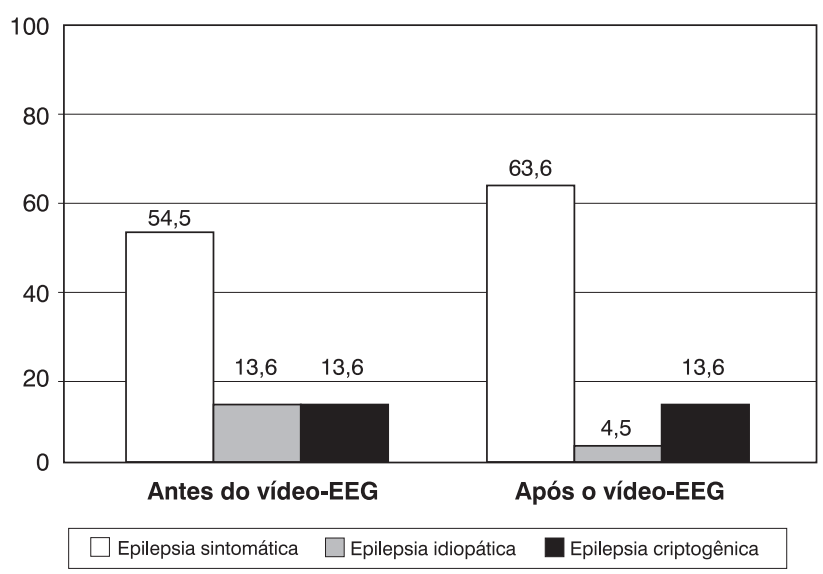

Figura 2 - Alteração da classificação das síndromes epilépticas, quanto à etiologia, após a monitorização 
Após o primeiro vídeo-EEG e a conseqüente mudança da terapêutica instituída, uma segunda monitorização foi requerida em cinco pacientes, para avaliar a resposta clínica ao novo tratamento.

\section{Grupo II}

Eventos clínicos foram registrados nos oito pacientes incluídos neste grupo, que apresentavam períodos de alteração comportamental repetitiva, por vezes acompanhados por movimentos repetitivos, sem etiologia definida. Quatro (50\%) destes apresentaram eventos epilépticos e, nestes, a mudança de diagnóstico levou à alteração da conduta terapêutica. Dois pacientes (25\%) apresentaram distúrbios do movimento, e dois (25\%), eventos não epilépticos (masturbação infantil e síncope), fortalecendo a suspeita diagnóstica prévia.

\section{Grupo III}

Oito crianças foram encaminhadas ao vídeo-EEG dia para diagnóstico diferencial entre declínio cognitivo e eventos subclínicos e/ou EME não convulsivo, eventos estes que não foram elucidados pelo EEG de rotina.

Em cinco pacientes (62,5\%), o quadro de declínio cognitivo foi decorrente de EME (estado de mal de ausência em quatro pacientes e estado de mal mioclônico em um). Três pacientes apresentaram eventos não epilépticos, caracterizados, respectivamente, por disautonomias, mioclonias não corticais e deterioração cognitiva decorrente da encefalopatia de base.

\section{Conseqüiências do vídeo-EEG dia na terapêutica e na investigação clínica}

A reclassificação quanto à natureza dos eventos paroxísticos não epilépticos em epilépticos ocorreu em seis pacientes (Figura 3), no grupo de 36 pacientes, que tiveram seus eventos monitorizados.

Modificações terapêuticas maiores foram realizadas em $21 / 38(55,3 \%)$ pacientes. Dez pacientes foram encaminhados para avaliação pré-operatória; dois tiveram as drogas antiepilépticas (DAE) retiradas; em nove, medicações foram introduzidas (DAE em cinco e drogas psicotrópicas em dois). Em nove pacientes, somente o ajuste nas doses dos medicamentos foi necessário. O vídeo-EEG dia foi útil na indicação de uma região para direcionamento do estudo de neuroimagem com RM em 10 pacientes, previamente classificados como tendo epilepsia criptogênica ou mesmo idiopática, e em outros dois com outras hipóteses diagnósticas (uma suspeita de desordem metabólica e outra suspeita de crises não epilépticas).

\section{Monitorização prolongada}

Dez pacientes foram encaminhados para o vídeo-EEG prolongado, oito por serem considerados candidatos à cirurgia e dois por apresentarem vídeo-EEGs dia inconclu-

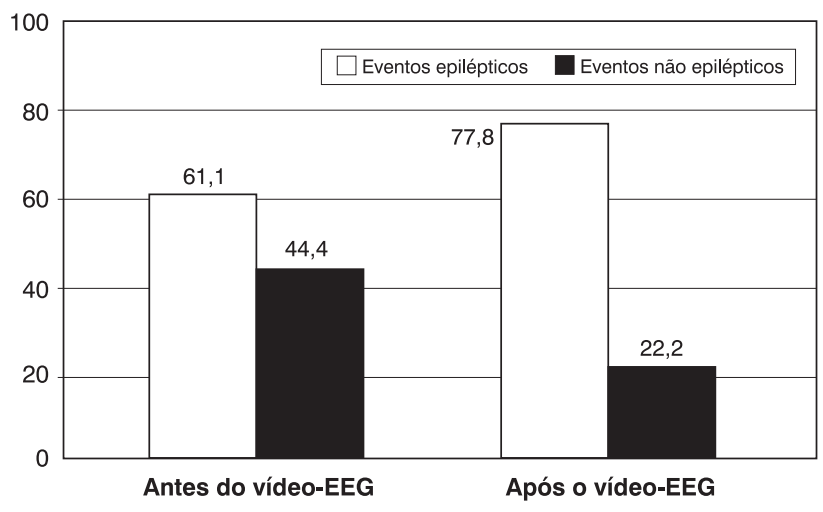

Figura 3 - Reclassificação dos eventos paroxísticos no grupo de 36 pacientes que apresentaram eventos clínicos durante a monitorização

sivos. Nestes pacientes, submetidos posteriormente ao vídeo-EEG prolongado, a hospitalização foi reduzida em virtude das informações previamente obtidas no vídeoEEG dia.

\section{Discussão}

A população pediátrica, em especial as crianças jovens e lactentes, representa o maior desafio para o diagnóstico dos eventos paroxísticos. Como salientado em outros estu$\operatorname{dos}^{5-7,14,15}$, a história fornecida pelos pais sobre as manifestações clínicas dos eventos paroxísticos, nesta faixa etária, é confusa e pouco elucidativa. Neste contexto, o vídeo-EEG passa a desempenhar um importante papel na elucidação diagnóstica e conduta terapêutica dos eventos paroxísticos da infância. Entretanto, o vídeo-EEG prolongado não é, na maioria das vezes, bem tolerado em crianças ${ }^{1,14-16}$, representando um fator estressor adicional às famílias que se vêem surpreendidas pelo aparecimento de eventos paroxísticos. Portanto, é importante estabelecer se este grupo de pacientes pode se beneficiar de uma monitorização mais breve na elucidação de eventos diagnósticos, ou se a limitação do tempo de estudo no vídeo-EEG dia ${ }^{16}$ significa um fator de inadequação do método. No nosso estudo, o vídeo-EEG dia foi elucidativo em $71 \%$ dos nossos casos no $1^{\circ}$ vídeo-EEG, similar aos encontrados por outros autores ${ }^{8,11}$, e passou para $94,7 \%$, considerando-se a $2^{\mathrm{a}}$ monitorização, independentemente da idade ou da queixa clínica. Acreditamos que estes índices elevados sejam decorrentes de vários fatores, tais como: um estudo realizado em um centro terciário, o uso de métodos de ativação individualizados (ex., alteração no ciclo circadiano) e a maior duração da monitorização, comparada à de outros ${ }^{4,10}$. Deve-se levar em conta ainda a população estudada, constituída por crianças, que se beneficiam deste tipo de monitorização, devido à frequiência elevada de crises epilépti- 
cas, quando comparada aos adultos com epilepsia refratária. Algumas estratégias podem ser adotadas para aumentar ainda mais a sensibilidade deste método, como o encaminhamento pelo clínico ou a orientação aos pais para a realização do exame em momentos em que haja uma piora na frequiência das crises e, como já anteriormente mencionado, a implementação de um segundo vídeo-EEG de 12 horas, quando necessário, medida esta que pode evitar a internação para um vídeo-EEG prolongado.

Uma das maiores indicações do vídeo-EEG é a classificação adequada da epilepsia para a melhor abordagem terapêutica. As crises generalizadas de início focal, freqüentes na infância ${ }^{5,6,17-19}$, representam um dos maiores desafios para o diagnóstico em crianças com epilepsia e, em nosso estudo, foram uma das principais razões de reclassificação das crises epilépticas. Conseqüentemente, este método permitiu, indiretamente, um melhor ajuste das DAE e direcionou os exames de neuroimagem em pacientes previamente diagnosticados como criptogênicos. A importância deste fato pode ser ilustrada pela mudança de conduta frente a esta reclassificação, que levou uma parte destes pacientes à intervenção cirúrgica precoce, impedindo o efeito deletério das crises epilépticas no cérebro em desenvolvimento.

O diagnóstico de eventos não epilépticos, em pacientes previamente tratados para epilepsia, impediu que estes pacientes recebessem DAE ou permitiu a sua retirada. Embora, como já salientado por outros ${ }^{20,21}$, a vantagem do vídeo-EEG dia nestes pacientes seja inegável, em nossa experiência constatamos a necessidade de tomar cuidado com o diagnóstico precipitado e a retirada brusca de medicações, visto que a comorbidade de epilepsia e eventos não epilépticos é alta. Assim, um manejo terapêutico baseado em uma avaliação breve pode levar a um aumento da morbidade.

Dentre as crianças, um grupo que particularmente se beneficiou da monitorização foi o das crianças com encefalopatias graves ou distúrbios psiquiátricos, que apresentam dificuldades para descrever sintomas subjetivos, dificultando, com isso, um diagnóstico clínico. No nosso estudo, o vídeo-EEG dia foi vital para o diagnóstico de epilepsia nestas crianças que apresentam um risco elevado de EME não convulsivo, difícil de ser detectado, devido ao contato pobre com o meio ${ }^{22-23}$. Neste ponto, o reconhecimento do evento é fundamental para a terapêutica apropriada.

Outros autores têm utilizado o vídeo-EEG dia para avaliação pré-operatória ${ }^{10}$, entretanto, nós temos a firme convicção que, para este objetivo, o vídeo-EEG prolongado é absolutamente necessário, devido à importância do detalhamento das crises epilépticas e do envolvimento de uma equipe multidisciplinar no planejamento do procedimento cirúrgico. Neste aspecto, o vídeo-EEG dia pode abreviar a monitorização pré-operatória, mas não deve substituir a monitorização prolongada.

Concluímos que, em nossa série, o vídeo-EEG dia estabeleceu o diagnóstico na maioria dos pacientes, corre- lacionando os dados clínicos com os eletrencefalográficos. Os registros foram bem tolerados pelas crianças, incluindo aquelas com doenças psiquiátricas. As principais vantagens do método foram a classificação das crises e síndromes epilépticas, o diagnóstico diferencial dos eventos não epilépticos e a detecção de crises epilépticas, imperceptíveis ao observador. A principal limitação, além do tempo de registro mais breve, reside na alta comorbidade da epilepsia com eventos não epilépticos, e, portanto, o registro de um evento não epiléptico não afasta por completo o diagnóstico de epilepsia, e a conduta deve ser criteriosa. Este estudo demonstra a utilidade e a importância do vídeo-EEG dia em pacientes pediátricos com eventos paroxísticos em que o EEG de rotina não foi capaz de elucidar o diagnóstico. Por outro lado, apesar do vídeo-EEG dia ser extremamente útil para reconhecimento de crises epilépticas e sua classificação, acreditamos que a monitorização prolongada seja o método de escolha para a avaliação pré-operatória de crianças candidatas à cirurgia de epilepsia.

\section{Referências bibliográficas}

1. Binnie CD, Rowan AJ, Overweg J, Meinardi H, Wisman T, Kamp A, et al. Telemetric EEG and video monitoring in epilepsy. Neurology 1981;31(3):298-303.

2. Mizrahi EM. Electroencephalographic/polygraphic/video monitoring in childhood epilepsy. J Pediatr 1984;105:1-9.

3. Lagerlund TD, Cascino GD, Cicora KM, Sharbrough FW. Long-term electroencephalographic monitoring for diagnosis and management of seizures. Mayo Clin Proc 1996;71(10): 1000-6.

4. Rowan AJ, Siegel M, Rosenbaum DH. Daytime intensive monitoring: comparison with prolonged intensive and ambulatory monitoring. Neurology 1987;37(3):481-4.

5. King DW, Dyken PR, Spinks IL Jr, Murvin AJ. Infantile spasms: ictal phenomena. Pediatr Neurol 1985;1(4):213-8.

6. Donat JF, Wright FS. Unusual variants of infantile spasms. J Child Neurol 1991;6(4):313-8.

7. Duchowny MS, Resnick TJ, Deray MJ, Alvarez LA. Video EEG diagnosis of repetitive behavior in early childhood and its relationship to seizures. Pediatr Neurol 1988;4(3):162-4.

8. Connolly MB, Wong PK, Karim Y, Smith S, Farrell K. Outpatient video-EEG monitoring in children. Epilepsia 1994;35(3):477-81.

9. Foley CM, Legido A, Miles DK, Grover WD. Diagnostic value of pediatric outpatient video-EEG. Pediatr Neurol 1995; 12(2): 120-4.

10. Al-Qudah AA, Abu-Sheik S, Tamimi AF. Diagnostic value of short duration outpatient video electroencephalographic monitoring. Pediatr Neurol 1999;21(3):622-5.

11. Chen LS, Mitchell WG, Horton EJ, Snead OC 3rd. Clinical utility of video-EEG monitoring. Pediatr Neurol 1995;12(3): 220-4.

12. Jasper HH. Report of the committee on methods of clinical examination in electroencephalography. Electroencephal Clin Neurophysiol 1958;10:370-5.

13. Commission on Classification and Terminology of the International League Against Epilepsy. Proposal for revisited clinical and electroencephalographic classification of epilepsy and epileptic syndromes. Epilepsia 1989;30:389-99. 
14. Duchowny MS. Intensive monitoring in the epileptic child. J Clin Neurophysiol 1985;2(3):203-19.

15. Duchowny MS. Complex partial seizures of infancy. Arch Neurol 1987;44(9):911-4.

16. Legatt AD, Ebersole JS. Options for long-term monitoring. In: Engel Jr J, Pedley TA, editores. Epilepsy: a comprehensive textbook. Filadélfia: Lippincott-Raven Publishers; 1998. p.1001-10.

17. Hamer HM, Wyllie E, Luders HO, Kotagal P, Acharya J. Symptomatology of epileptic seizures in the first three years of life. Epilepsia 1999;40(7):837-44.

18. Dravet C, Catani C, Bureau M, Roger J. Partial epilepsies in infancy: a study of 40 cases. Epilepsia 1989;30(6):807-12.

19. Yamamoto N, Watanabe K, Negoro T, Takaesu E, Aso K, Furune $\mathrm{S}$, et al. Complex partial seizures in children: ictal manifestations and their relation to clinical course. Neurology 1987;37(8): 1379-82.

20. McGonigal A, Oto M, Russell AJ, Greene J, Duncan R. Outpatient video EEG recording in the diagnosis of non-epileptic seizures: a randomised controlled trial of simple suggestion techniques. $\mathbf{J}$ Neurol Neurosurg Psychiatry 2002;72(4):549-51.

21. Bhatia M, Sinha PK, Jain S, Padma MV, Maheshwari MC. Usefulness of short-term video EEG recording with saline induction in pseudoseizures. Acta Neurol Scand 1997;95(6): 363-6.
22. Matsumoto A, Kumagai T, Miura K, Miyazaki S, Hayakawa C, Yamanaka T. Epilepsy in Angelman syndrome associated with chromosome 15q deletion. Epilepsia 1992;33(6):1083-90.

23. Sugimoto T, Yasuhara A, Ohta T, Nishida N, Saitoh S, Hamabe $\mathrm{J}$, et al. Angelman syndrome in three siblings: characteristic epileptic seizures and EEG abnormalities. Epilepsia 1992; 33(6):1078-82.

Endereço para correspondência:

Dra. Kette D. Valente

Laboratório de Neurofisiologia Clínica da Faculdade de Medicina da Universidade de São Paulo

Rua Jesuíno Arruda, 901 - ap. 51

CEP 04532-082 - São Paulo, SP

Tels.: (11) 3069.6518 / 3079.5493 - Fax: (11) 3079.5493

E-mail:kettevalente@msn.com 